\title{
Resolved-sideband cooling and measurement of a micromechanical oscillator close to the quantum limit
}

\author{
A. Schliesser,,$* *$ O. Arcizet,,$* *$ R. Rivière,,$* *$ and T.J. Kippenberg ${ }^{1,2,+}$ \\ ${ }^{1}$ Max Planck Institut für Quantenoptik, 85748 Garching, Germany \\ ${ }^{2}$ École Polytechnique fédérale de Lausanne (EPFL), CH-1015 Lausanne, Switzerland
}

(Dated: October 16, 2018) 


\begin{abstract}
The observation of quantum phenomena in macroscopic mechanical oscillators [1, 2] has been a subject of interest since the inception of quantum mechanics. It may provide insights into the quantum-classical boundary, experimental investigation of the theory of quantum measurements [1, 3, 4], the origin of mechanical decoherence [5] and generation of non-classical states of motion. Prerequisite to this regime are both preparation of the mechanical oscillator at low phonon occupancy and a measurement sensitivity at the scale of the spread $\Delta x$ of the oscillator's ground state wavefunction. Over the past decade, it has been widely perceived that the most promising approach to address these two challenges are electro nanomechanical systems [2, 6, 7, ㅇ, 9, 10], which can be cooled with milli-Kelvin scale dilution refrigerators, and feature large $\Delta x \sim 10^{-14} \mathrm{~m}$ resolvable with electronic transducers such as a superconducting single-electron transistor [7, 8, 11], a microwave stripline cavity $[9]$ or a quantum interference device [12]. In this manner, thermal occupation as low as 25 quanta [7, 10] has been measured. Here we approach for the first time the quantum regime with a mechanical oscillator of mesoscopic dimensions-discernible to the bare eye-and 1000-times more massive than the heaviest nano-mechanical oscillators used to date. Imperative to these advances are two key principles of cavity optomechanics [13]: Optical interferometric measurement of mechanical displacement at the attometer level [14, 15], and the ability to use measurement induced dynamic back-action [16, 17, 18, 19] to achieve resolved sideband laser cooling [9, 20] of the mechanical degree of freedom. Using only modest cryogenic pre-cooling to $1.65 \mathrm{~K}$, preparation of a mechanical oscillator close to its quantum ground state ( $63 \pm 20$ phonons) is demonstrated. Simultaneously, a readout sensitivity that is within a factor of $5.5 \pm 1.5$ of the standard quantum limit [1, 21] is achieved. Taking measurement backaction into account, this represents the closest approach to the Heisenberg uncertainty relation for continuous position measurements yet demonstrated. The reported experiments mark a paradigm shift in the approach to the quantum limit of mechanical oscillators using optical techniques and represent a first step into a new era of experimental investigation which probes the quantum nature of the most tangible harmonic oscillator: a mechanical vibration.
\end{abstract}

*These authors contributed equally to this work.

tobias.kippenberg@epfl.ch 
The experimental setting of the present work is a cavity optomechanical system, which parametrically couples optical and mechanical degrees of freedom via radiation pressure. In the present case, toroidal microresonators are employed which exhibit (cf. Fig. 1) strong, inherent opto-mechanical coupling between high-quality factor $\left(Q>10^{8}\right)$ optical whispering gallery modes and the mechanical radial breathing mode [22] (RBM), featuring high frequency (65 and $122 \mathrm{MHz}$ for the resonators used in this work), and effective masses [23] on the order of 1-10 ng (cf. Fig. 1b). The quality factors of the RBM can reach values up to 80,000 if clamping losses are mitigated by modal engineering [24]. To achieve a regime of low mechanical oscillator occupancy we apply laser cooling to a cryogenically pre-cooled micromechanical oscillator with high frequency. Figure 1 shows a schematic of the experiment. A chip with micro-resonators is inserted into a Helium exchange gas cryostat. Piezoelectric actuators enable positioning of a tapered optical fiber used for evanescent coupling with a resolution sufficient to adjust the taper-toroid gap to critical coupling. The total optical loss through the cryogenic environment can reach values below 25\%. Low pressure (0.1-50 mbar) Helium exchange gas is admitted into the sample chamber, thermalizing the sample with a heat exchanger through which ${ }^{4} \mathrm{He}$ is pumped from a reservoir of liquid ${ }^{4} \mathrm{He}$. An exchange gas temperature of $1.65 \mathrm{~K}$ is achieved. Due to the low heat conductivity of glass, and possible light absorption, it is of prime importance to verify the thermalization of the mechanical oscillator to $1.65 \mathrm{~K}$. To this end, we perform noise thermometry using the RBM. A low power $(<2 \mu \mathrm{W})$ laser is tuned into resonance with a high- $Q$ optical mode. Fluctuations of the cavity radius-as induced by thermal excitation of the RBM-induce resonance frequency fluctuations of the cavity, which are imprinted as phase fluctuations on the laser light coupling back to the tapered fiber (cf. Figure 1c). A phase-sensitive detection scheme enables measurement of the Lorentzian displacement noise spectrum of the thermal (Brownian) motion of the RBM, characterized by its resonance frequency $\Omega_{\mathrm{m}}$, mechanical damping rate $\Gamma_{\mathrm{m}}$ and peak displacement amplitude $S_{x x}^{\text {th }}\left(\Omega_{\mathrm{m}}\right)$ (cf. Methods summary). Figure 2a shows the resulting mechanical mode temperature as derived via the equipartition theorem from the independently calibrated noise spectra, where phase-sensitive detection was accomplished using the Pound-Drever-Hall technique. Importantly, the temperature of the sample follows the exchange gas, demonstrating that excellent thermalization is achieved, a key prerequisite for the experiments described from here on. For the $62 \mathrm{MHz}$ sample thermalization to 1.65 $\mathrm{K}$ entails an initial average occupancy of $\langle n\rangle=k_{\mathrm{B}} T_{\mathrm{RBM}} / \hbar \Omega_{\mathrm{m}} \approx 560$, while for the $122 \mathrm{MHz}$ 
sample a low occupancy of $\langle n\rangle \approx 280$ is attained. Note that despite the modest pre-cooling to $1.65 \mathrm{~K}$ these occupancies are identical to those of a $1 \mathrm{MHz}$ nano-mechanical oscillator thermalized to a dilution refrigerator temperature below $20 \mathrm{mK}$, emphasizing the significant advantage of working with high frequency oscillators.

Measuring the mechanical displacement associated with such a massive oscillator at low occupancies requires high sensitivity, in particular, since the mechanical quality factor of silica is reduced to $\sim 2000$ at $1.65 \mathrm{~K}$ due to losses originating from phonon coupling to structural defect states [25] (note that damping by the exchange gas is negligible, and that the mechanical $\mathrm{Q}$ factor improves again at lower temperatures [26]). The required attometer-level sensitivity can (so far) only be achieved with optical transducers. Following our previous work, we employ homodyne spectroscopy [15] based on a quantum-noise limited titanium sapphire laser (in both amplitude and phase), which is resonantly coupled to WGM resonance in the vicinity of a wavelength of $780 \mathrm{~nm}$. The laser's phase shift introduced by the mechanical fluctuations are detected interferometrically, by comparison with a high-power (2-5 mW) optical phase reference (local oscillator). Frequency analysis of this signal yields the thermal noise displacement spectrum $S_{x x}^{\text {th }}(\Omega)$, on top of a measurement background. Figure $2 \mathrm{~b}$ shows data obtained from the RBM of a $55 \mu \mathrm{m}$-diameter microresonator cooled to $\mathrm{T}=2.4 \mathrm{~K}$, or $\langle n\rangle \approx 770$. The background of this measurement is at a level of $1.5 \cdot 10^{-18} \mathrm{~m} / \sqrt{\mathrm{Hz}}$, which is only a factor of $5.5 \pm 1.5$-times higher than the standard quantum limit (SQL) [1, 21], given by $\sqrt{S_{x x}^{\mathrm{SQL}}\left(\Omega_{\mathrm{m}}\right)}=\sqrt{\hbar / m_{\mathrm{eff}} \Gamma_{\mathrm{m}} \Omega_{\mathrm{m}}}$ for a measurement at the mechanical resonance. This proves the counterintuitive notion, that measurements close to the standard quantum limit are possible, in spite of the here used strategy of using high frequency and comparatively massive oscillators (in contrast to nanomechanical systems).

In order to further decrease the number of thermal quanta of the mechanical oscillator we use cooling via radiation pressure dynamical backaction as predicted [16, 27] and recently experimentally demonstrated [17, 18, 19]. Similar to the atomic physics [28] case, ground state cooling requires accessing the resolved sideband regime [20, 29, 30], which necessitates the mechanical oscillator frequency to exceed the cavity decay rate (i.e. $\left.\Omega_{\mathrm{m}} \gg \kappa\right)$. This regime is moreover prerequisite for schemes such as two transducer quantum non-demolition (QND) measurements [1, 31] or the preparation of a mechanical oscillator in a squeezed state of motion [32]. Operation in the RSB regime as demonstrated in [10, 20] is accomplished using a cavity with a narrow resonance $(5.5 \mathrm{MHz}$ intrinsic decay rate and $9 \mathrm{MHz}$ mode 
splitting), which is broadened to a $\kappa / 2 \pi \approx 19 \mathrm{MHz}$-wide resonance due to fiber coupling (corresponding to a loaded finesse of $\sim 70,000$ ). The laser is subsequently tuned to the lower mechanical sideband, i.e. red-detuned by $65.2 \mathrm{MHz}$, the resonance frequency of this sample's RBM. For this detuning the circulating power is reduced by a factor of $4 \Omega_{\mathrm{m}}^{2} / \kappa^{2}$. At the same time, the sensitivity to mechanical displacements is slightly reduced. In the ideal case of a highly overcoupled cavity, with unity detection efficiency and no excess noise except for the laser's intrinsic quantum noise, the imprecision noise spectral density, i.e. the background of the measurement caused by detection shot noise, is given by:

$$
S_{x x}(\Omega)=\frac{\hbar \omega}{16 g_{0}^{2} \cdot P}\left(\frac{\Delta^{2}+(\kappa / 2)^{2}}{\kappa / 2}\right)^{2}\left(1+\Omega^{2} \frac{\Omega^{2}+(\kappa / 2)^{2}-2 \Delta^{2}}{\left(\Delta^{2}+(\kappa / 2)^{2}\right)^{2}+\Omega^{2}(\kappa / 2)^{2}}\right)
$$

where the opto-mechanical coupling $g_{0}=d \omega / d x$, and $d \omega / d x=\omega / R$ in the present embodiment. Moreover, $R$ is the cavity radius, $P$ the launched input laser power, $\omega / 2 \pi$ the optical resonance frequency, $\Delta / 2 \pi$ the detuning from the cavity resonance, and $\Omega / 2 \pi$ the analysis frequency. In the resolved sideband case $\Omega_{\mathrm{m}} \gg \kappa$, this expression simplifies to $S_{x x}\left(\Omega_{\mathrm{m}}\right)=\hbar \omega \Omega_{\mathrm{m}}^{2} / 4 g_{0}^{2} P$ at the mechanical resonance frequency, when detuned to the first (upper or lower) mechanical sideband $|\Delta|=\Omega_{\mathrm{m}}$. This is only a factor of 4 higher than in the resonant readout case $\Delta=0$. We note the interesting result that this expression does not depend on optical finesse in the deeply resolved sideband regime.

As shown in previous work [10, 20], the laser detuned to the lower sideband leads to a significant reduction of the thermal occupation (i. e. cooling), as evidenced by the reduced area underneath the peaks associated with the oscillator's thermal noise (cf. Fig. 3b). The underlying physical mechanism giving rise to cooling is enhanced anti-Stokes scattering into the cavity mode, whereby each scattering process annihilates a thermal phonon. In the resolved-sideband regime, ground state cooling is possible in principle, and the minimum occupation that can be reached is given by $\langle\tilde{n}\rangle \cong \kappa^{2} / 16 \Omega_{\mathrm{m}}^{2} \ll 1[29$, 30]. As the laser cools the resonator out of equilibrium with the thermal bath (at temperature $T$ ), however, heating through the bath competes with laser cooling and leads to a final occupation of $\left\langle n_{\mathrm{f}}\right\rangle \approx \frac{\Gamma_{\mathrm{m}}}{\Gamma_{\mathrm{m}}+\Gamma_{\text {cool }}} k_{\mathrm{B}} T / \hbar \Omega_{\mathrm{m}}$, where $\Gamma_{\mathrm{m}}$ is the intrinsic damping rate and $\Gamma_{\text {cool }}$ the laser induced cooling rate. In the case of the data shown in Fig. 3, a strong increase in the damping with a concomitant reduction of the thermal occupation can be observed. The highest attained total damping rate is $\left(\Gamma_{\mathrm{m}}+\Gamma_{\text {cool }}\right) / 2 \pi=370 \mathrm{kHz}$, reached with a launched power of $\sim 0.2 \mathrm{~mW}$. Evaluation of the calibrated thermal noise spectrum reveals an effective mode 
temperature of $200 \pm 60 \mathrm{mK}$, which corresponds to an average occupation as low as $\left\langle n_{\mathrm{f}}\right\rangle=$ $63 \pm 20$ quanta. This is the lowest reported occupancy for a cavity optomechanical cooling experiment reported to date; slightly lower occupancy, $\left\langle n_{\mathrm{f}}\right\rangle=25$, has only been attained in the context of conventional dilution refrigeration of nanomechanical oscillators [7], albeit with a signal-to-background ratio well below unity. Back-action cooling techniques applied to nano-mechanical oscillators at milli-Kelvin temperatures achieve occupation numbers about one order of magnitude higher [7, 10].

Moreover, a key aspect of the reported experiments is operation in the resolved sideband regime. To illustrate the instrumental role of the RSB regime for accessing low phonon occupation number of mechanical oscillators, we compare the cooling run just described with a further, independent run with a smaller sample $\left(\Omega_{\mathrm{m}} / 2 \pi=122 \mathrm{MHz}\right)$, exhibiting significantly broader linewidth (fiber coupling broadened to $\kappa / 2 \pi=155 \mathrm{MHz}$ ), as shown in Fig. 4. In this case, a significant deviation from the linear cooling behavior is observed, with higher temperatures measured than expected based on the cooling rate. It is possible to model this deviation by taking an intracavity-power dependent heating of the mechanical mode into account. Heating of the mechanical mode can be caused by both quantum [1, 33] or classical fluctuations [20] of the radiation pressure force. While the latter is ruled out in our experiment by the use of a low-noise laser source (quantum limited in amplitude and phase for the Fourier frequency of interest), the former effect cannot account for the heating due to its much smaller magnitude, as detailed below. Instead, the effect is attributed to heating via laser absorption. Both data sets exhibit heating of ca. $10 \mathrm{~K} /$ Watt of circulating optical power. While quantitative modeling would have to take characteristics of the optical mode and heat transfer in the gas-cooled sample into account, we note that similar values of laser induced heating were extracted from studies of the optical bistability at low temperature at a wavelength of $1.5 \mu \mathrm{m}$ [25]. Indeed, operating in the resolved-sideband regime [20] allows to mitigate this effect and enables the results demonstrated in this work, as well as future work aiming at achieving ground-state cooling, as it strongly reduces the circulating intracavity power.

It is also interesting to consider the reported measurements from the perspective of the theory of quantum measurement [1, 34]. For linear continuous measurements as those demonstrated here, the total measurement uncertainty arises from two intrinsic sources of noise: measurement imprecision and measurement backaction. Measurement imprecision 
arises from fluctuations at the output of the measurement device, which are not related to mechanical oscillator motion. In the case of an optical interferometric measurement as reported here, measurement imprecision can be reduced to the shot noise in the detection process (cf. eq. (1)). Measurement backaction, on the other hand, describes the perturbation of the mechanical oscillator by the process of the measurement. For a mechanical oscillator, this occurs in the form of a fluctuating force, characterized by a spectral density $S_{F F}(\Omega)$. Quantum mechanics poses strict limits on how small $S_{x x}(\Omega)$ and $S_{F F}(\Omega)$ can be; the product obeying $\sqrt{S_{x x}(\Omega) \cdot S_{F F}(\Omega)} \geq \hbar / 2$ where an equality can be reached in the ideal case and in the absence of correlations of imprecision and backaction noise [1]. While classical fluctuations (caused by laser heating or classical laser amplitude and phase noise) can increase the value of the measurement backaction, the lowest possible force noise for a coherent input is given by the quantum backaction (QBA):

$$
S_{F F}^{\mathrm{qba}}(\Omega)=\frac{\hbar}{2 \omega} g_{0}^{2} P_{\text {in }}\left(\frac{\kappa^{2}}{(\kappa / 2)^{2}+\Delta^{2}}\right)\left(\frac{1}{(\kappa / 2)^{2}+\left(\Delta-\Omega_{\mathrm{m}}\right)^{2}}+\frac{1}{(\kappa / 2)^{2}+\left(\Delta+\Omega_{\mathrm{m}}\right)^{2}}\right)
$$

where $S_{F F}^{\mathrm{qba}}\left(\Omega_{\mathrm{m}}\right)=2 g_{0}^{2} P_{\mathrm{in}} \hbar / \omega \Omega_{\mathrm{m}}^{2}$ in the deeply resolved sideband regime $\left(|\Delta|=\Omega_{\mathrm{m}} \gg \kappa\right)$. For the parameters of the measurement with $\left\langle n_{\mathrm{f}}\right\rangle=63 \pm 20$, we find $\sqrt{S_{F F}^{\text {qba }}\left(\Omega_{\mathrm{m}}\right)} \approx 1 \mathrm{fN} / \sqrt{\mathrm{Hz}}$. For the present experiments, the dominant measurement backaction effect is the heating by laser absorption (as observed in Fig. 4), which increases the magnitude of the thermal Langevin force. Indeed, using the effective mechanical susceptibility (at resonance) of the laser-cooled oscillator $\left|\chi_{\text {eff }}\left(\Omega_{\mathrm{m}}\right)\right|=\left(m_{\mathrm{eff}}\left(\Gamma_{\mathrm{m}}+\Gamma_{\text {cool }}\right) \Omega_{\mathrm{m}}\right)^{-1}$, we can retrieve the magnitude of the total force spectral density from the measurement of its displacement spectrum via $S_{F F}^{\text {tot }}\left(\Omega_{\mathrm{m}}\right)=S_{x x}^{\text {th }}\left(\Omega_{\mathrm{m}}\right) /\left|\chi_{\text {eff }}\left(\Omega_{\mathrm{m}}\right)\right|^{2}$. It amounts to $\sqrt{S_{F F}^{\text {tot }}\left(\Omega_{\mathrm{m}}\right)} \approx 8 \mathrm{fN} / \sqrt{\mathrm{Hz}}$ and is dominated by the thermal Langevin force associated with the toroid's temperature. While only a part of this force is caused by the measurement process via the heating induced temperature increase, we can nonetheless calculate the product $\sqrt{S_{x x}\left(\Omega_{\mathrm{m}}\right) \cdot S_{F F}^{\text {tot }}\left(\Omega_{\mathrm{m}}\right)}$ as an upper bound, of how closely the ideal case has been approached in these measurements. For this the conservative assumption that the entire force spectral density acting on the mechanical oscillator (including the thermal Langevin force) is caused by measurement backaction is used. Using $\sqrt{S_{x x}(\Omega)} \approx 1.4 \cdot 10^{-18} \mathrm{~m} / \sqrt{\mathrm{Hz}}$ we thereby find a product of $\sqrt{S_{x x}\left(\Omega_{\mathrm{m}}\right) \cdot S_{F F}^{\text {tot }}\left(\Omega_{\mathrm{m}}\right)}$ as low as $230 \cdot \hbar / 2$. This represents a factor of 4 improvement compared to the closest approach made with a nano-electromechanical system [7, 34]. We expect that improvements on our results are readily attainable by operating more deeply in the resolved-sideband 
regime [20], in a colder cryogenic environment (such as ${ }^{3} \mathrm{He}$ ), and with reduced mechanical dissipation by employing e.g. crystalline resonators [35]. The properties of the system demonstrated here-a massive oscillator, prepared with very low average occupation, which is coupled to an ultra low loss fiber transport medium is pivotal for a variety of experiments and protocols involving photons and phonons, such as radiation pressure squeezing [36, 37], entanglement [38] or QND measurements [32, 39].

In summary, our result signal a paradigm shift in the access to the quantum regime of mechanical oscillators by demonstrating the clear feasibility to enter this regime using significantly more massive cavity optomechanical [13, 40] systems and using modest precooling; a regime so far only attained with nanomechanical oscillator thermalized to milliKelvin scale dilution refridgerator temperatures. As such, the reported experiments-which demonstrate preparation and sensitive readout of mechanical oscillator with few quantamark a first step into a new era of experimental investigation of mechanical systems in the quantum regime, which has applications ranging from fundamental predictions of quantum measurement theory tests of quantum mechanics to the generation of non-classical states of motion and study of mechanical decoherence.

\section{Acknowledgments}

This work was supported by an Independent Max Planck Junior Research Group of the Max Planck Society, the Deutsche Forschungsgemeinschaft (DFG-GSC) and a Marie Curie Excellence Grant, and the FP7 project "MINOS". O. A. acknowledges funding from a Marie Curie Grant (project QUOM). Thomas Becker is gratefully acknowledged for support with the cryogenic experiments, and Jörg Kotthaus for sample fabrication.

[1] V. B. Braginsky and F. Y. Khalili, Quantum Measurement (Cambridge University Press, 1992).

[2] K. C. Schwab and M. L. Roukes, Physics Today 58, 36 (2005).

[3] S. Bose, K. Jacobs, and P. L. Knight, Physical Review A 59, 3204 (1999).

[4] I. Tittonen, G. Breitenbach, T. Kalkbrenner, T. Müller, R. Conradt, S. Schiller, E. Steinsland, N. Blanc, and N. F. de Rooij, Physical Review A 59, 1038 (1999). 
[5] W. Marshall, C. Simon, R. Penrose, and D. Bouwmeester, Physical Review Letters 91, 130401 (2003).

[6] A. Cleland and M. Roukes, Nature 392, 160 (1998).

[7] A. Naik, O. Buu, M. D. LaHaye, A. D. Armour, A. A. Clerk, M. P. Blencowe, and K. C. Schwab, Nature 443, 193 (2006).

[8] M. D. LaHaye, O. Buu, B. Camarota, and K. C. Schwab, Science 304, 74 (2004).

[9] C. A. Regal, J. D. Teufel, and K. W. Lehnert, Nature Physics 4, 555 (2008).

[10] J. D. Teufel, J. D. Harlow, C. A. Regal, and K. W. Lehnert, Physical Review Letters 101, 197203 (2008).

[11] R. G. Knobel and A. N. Cleland, Nature 424, 291 (2003).

[12] S. Etaki, M. Poot, I. Mahbobb, K. Onomitsu, H. Yamaguchi, and H. S. J. van der Zant, Nature Physics 4, 785 (2008).

[13] T. J. Kippenberg and K. J. Vahala, Science 321, 1172 (2008).

[14] O. Arcizet, P.-F. Cohadon, T. Briant, M. Pinard, A. Heidmann, J.-M. Mackowski, C. Michel, L. Pinard, O. Francais, and L. Rousseau, Physical Review Letters 97, 133601 (2006).

[15] A. Schliesser, G. Anetsberger, R. Rivière, O. Arcizet, and T. J. Kippenberg, New Journal of Physics 10, 095015 (2008).

[16] M. I. Dykman, Sov. Phys. Solid State 20, 1306 (1978).

[17] S. Gigan, H. R. Böhm, M. Paternosto, F. Blaser, G. Langer, J. B. Hertzberg, K. C. Schwab, D. Bäuerle, M. Aspelmeyer, and A. Zeilinger, Nature 444, 67 (2006).

[18] O. Arcizet, P.-F. Cohadon, T. Briant, M. Pinard, and A. Heidmann, Nature 444, 71 (2006).

[19] A. Schliesser, P. Del'Haye, N. Nooshi, K. Vahala, and T. Kippenberg, Physical Review Letters 97, 243905 (2006).

[20] A. Schliesser, R. Rivière, G. Anetsberger, O. Arcizet, and T. Kippenberg, Nature Physics 4, 415 (2008).

[21] C. M. Caves, Physical Review D 23, 1693 (1981).

[22] T. J. Kippenberg, H. Rokhsari, T. Carmon, A. Scherer, and K. J. Vahala, Physical Review Letters 95, 033901 (2005).

[23] M. Pinard, Y. Hadjar, and A. Heimann, European Physics Journal D 7, 107 (1999).

[24] G. Anetsberger, R. Rivière, A. Schliesser, O. Arcizet, and T. J. Kippenberg, Nature Photonics 2, 627 (2008). 
[25] O. Arcizet, R. Rivière, A. Schliesser, and T. J. Kippenberg, arxiv:0901.1292 (2009).

[26] R. O. Pohl, X. Liu, and E. Thompson, Review of Modern Physics 74, 991 (2002).

[27] V. B. Braginsky and S. P. Vyatchanin, Physics Letters A 293, 228 (2002).

[28] D. J. Wineland and W. M. Itano, Physical Review A 20, 1521 (1979).

[29] I. Wilson-Rae, N. Nooshi, W. Zwerger, and T. J. Kippenberg, Physical Review Letters 99, 093901 (2007).

[30] F. Marquardt, J. P. Chen, A. A. Clerk, and S. M. Girvin, Physical Review Letters 99, 093902 (2007).

[31] V. B. Braginsky and F. Y. Khalili, Reviews of Modern Physics 68, 1 (1996).

[32] A. A. Clerk, F. Marquardt, and K. Jacobs, New Journal of Physics 10, 095010 (2008).

[33] C. Genes, D. Vitali, P. Tombesi, S. Gigan, and M. Aspelmeyer, Physical Review A 77, 033804 (2008).

[34] A. A. Clerk, M. H. Devoret, S. M. Girvin, F. Marquardt, and R. J. Schoelkopf, arXiv:0810.4729 (2008).

[35] V. S. Ilchenko, A. A. Savchenkov, A. B. Matsko, and L. Maleki, Physical Review Letters 92, $043903(2004)$

[36] C. Fabre, M. Pinard, S. Bourzeix, A. Heidmann, E. Giacobino, and S. Reynaud, Physical Review A 49, 1337 (1994).

[37] S. Mancini and P. Tombesi, Physical Review A 49, 4055 (1994).

[38] D. Vitali, S. Gigan, A. Ferreira, H. R. Bohm, P. Tombesi, A. Guerreiro, V. Vedral, A. Zeilinger, and M. Aspelmeyer, Physical Review Letters 98, 030405 (2007).

[39] A. Heidmann, Y. Hadjar, and M. Pinard, Applied Physics B 64, 173 (1997).

[40] T. J. Kippenberg and K. Vahala, Optics Express 15, 17172 (2007). 

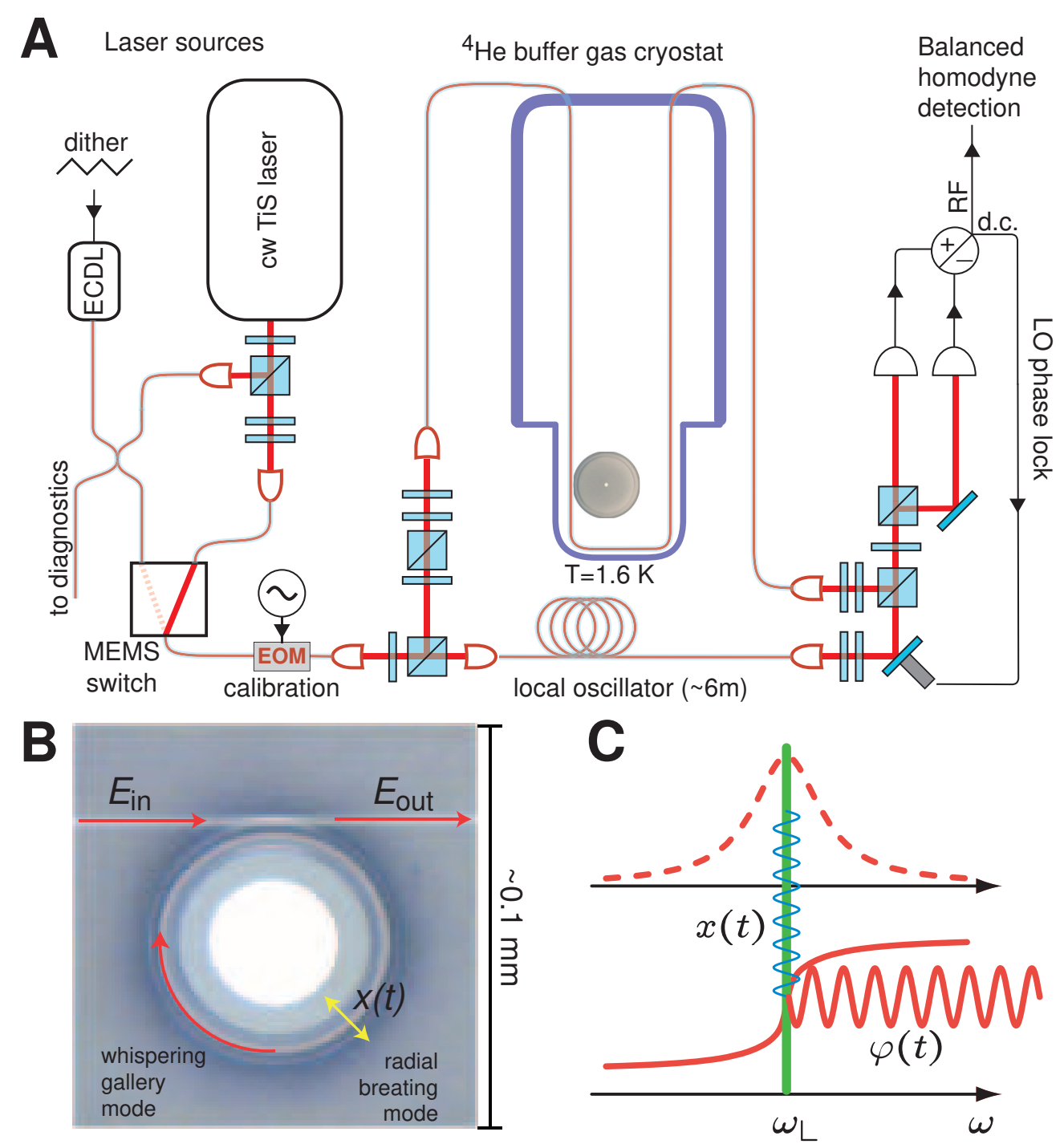

FIG. 1: Cryogenic cooling and displacement measurements of a micromechanical oscillator. (A to C) A silica microtoroid is held in a $1.65 \mathrm{~K}$-cold ${ }^{4} \mathrm{He}$ atmosphere. The toroid supports both high-Q optical whispering gallery modes and a mechanical radial breathing mode, which are parametrically coupled to an optical resonance frequency shift induced by mechanical displacement. High-Q optical resonances are identified using a tunable external-cavity diode laser (ECDL). To probe the mechanical oscillator, the light input is switched to a low-noise Ti:Sapphire. A small fraction of the laser beam is sent into the cryostat and couples to the WGM by evanescent coupling from a fiber taper approached to the rim of the toroid. Balanced homodyne measurement of the laser phase as transmitted through the taper is implemented using a Mach-Zehnder fiber interferometer (phase plates and polarizing beam splitters are only schematically indicated). A modulation $x(t)$ of the radius of the cavity $(\mathrm{C})$ induces a modulation of the phase of the light $\phi(t)$ emerging from the cavity. This phase shift is detected by comparison with a phase reference, derived from the same laser in a beam splitter followed by a balanced detector. 

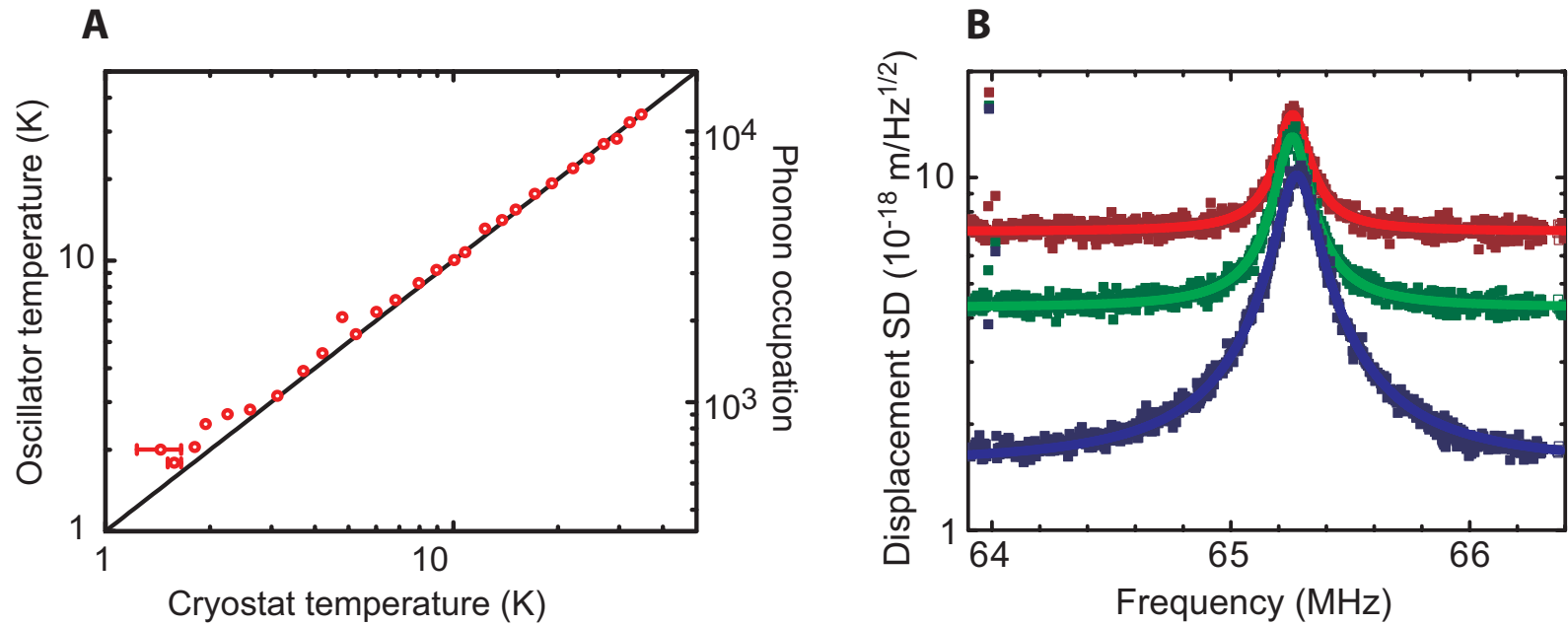

FIG. 2: Thermalization and probing of a micromechanical oscillator (A) Using noise thermometry, the temperature of the mechanical oscillator is determined as a function of the temperature of the cryostat (exchange gas). The noise temperature of the $62 \mathrm{MHz}$ mode follows the cryostat temperature in a linear manner, down to an occupation of less than 1000 phonons. (B) In spite of the low phonon occupation (770 in the case of this $65.3 \mathrm{MHz}$ oscillator) displacement monitoring with high signal-to-noise ratio is possible using optical techniques (at the attometer level in the present case). The optical power used to interrogate the mechanical oscillator was ca. $3 \mu \mathrm{W}$ (red trace), $10 \mu \mathrm{W}$ (green trace) and $100 \mu \mathrm{W}$ (blue trace). The measurement background is given by shot noise in the detection. 
A

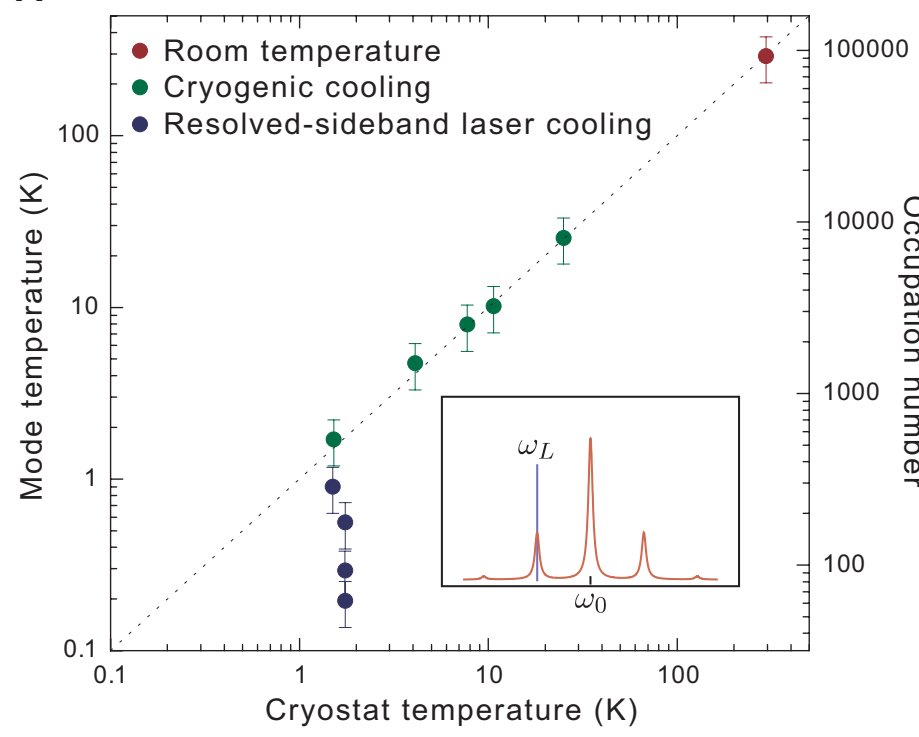

B

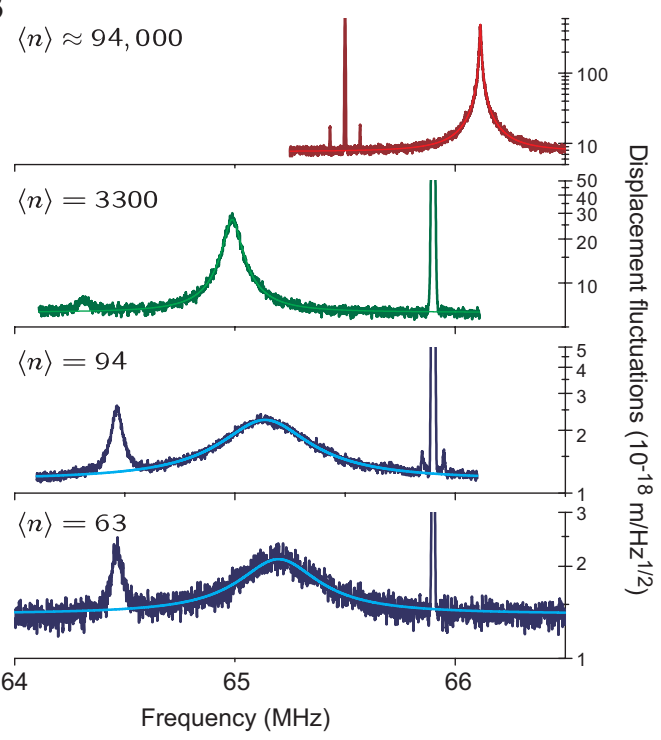

FIG. 3: Cryogenic precooling and resolved-sideband laser cooling. (A) Combined with pre-cooling in the cryostat to $1.65 \mathrm{~K}$, resolved-sideband cooling reduces the occupation of the oscillator further to $\left\langle n_{\mathrm{f}}\right\rangle=63 \pm 20$ phonons. The inset illustrates the laser (blue line) detuned to the lower mechanical sideband of the cavity spectrum (red line). (B) Displacement noise spectra of the RBM with four different average occupation numbers, together with Lorentzian fit, at room temperature (red curve) after cryogenic cooling to 10K (green curve), and after additional resolved sideband cooling (blue curves). The sharp calibration peak, and a second mode at slightly lower frequency are also shown. 

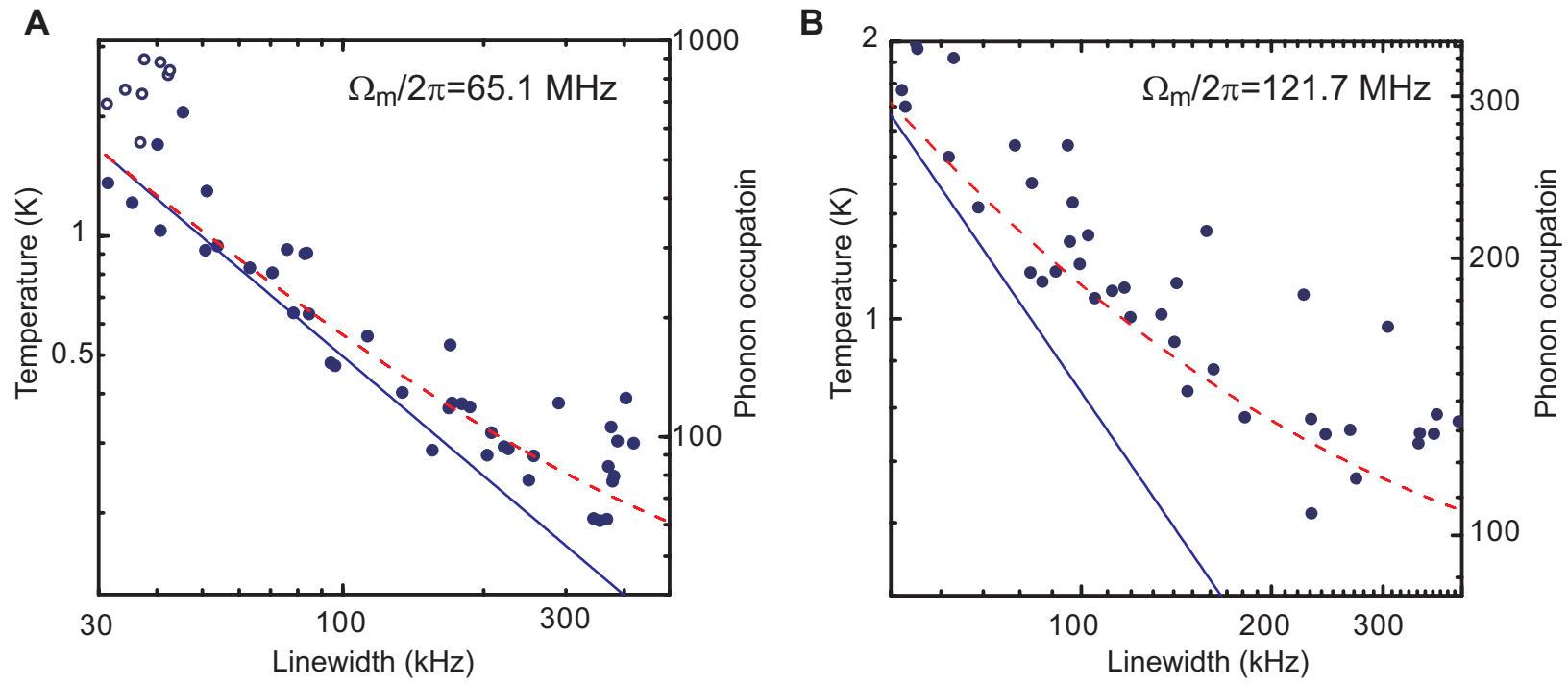

FIG. 4: Resolved sideband cooling of (A) a RBM at $65.1 \mathrm{MHz}$ and (B) an RBM at $121.7 \mathrm{MHz}$ frequency. The mode temperature and corresponding phonon occupation are reduced as the detuned laser induces additional damping, and therefore increases the linewidth of the thermal noise spectrum. Full points correspond to mechanical spectra taken with a detuned cooling laser, open points in (A) correspond to measurements with the laser tuned close to the optical resonance. The deviation from a linear cooling behaviour (blue line) indicates a backaction heating effect, which is compatible with heating of the structure originating from residual absorption (red dashed line). This effect is suppressed in the resolved sideband regime (panel (A)). 